\title{
GRB 130427A afterglow: a test for GRB models
}

\section{Massimiliano De Pasquale ${ }^{* \dagger}$}

Department of Astronomy and Space Sciences, Istanbul University, Beyazlt, 34119, Istanbul, Turkey

E-mail: m.depasquale@ucl.ac.uk

\section{J. Page}

Mullard Space Science Laboratory, University College London, Dorking RH56NT, United Kingdom

\section{A. Kann}

Istituto de Astrofisica de Andalucia (CSIC), Glorieta de Astronomia, E-18008 Granada, Spain

\section{S. R. Oates}

Department of Physics, University of Warwick, Coventry, CV4 7AL, UK

\section{S. Schulze}

Department of Particle Physics and Astrophysics, Weizmann Institute of Science, Rehovot 7610001, Israel

\section{B. Zhang}

Department of Physics and Astronomy, University of Nevada, Las Vegas, NV 89154, USA

\section{Z. Cano}

Instituto de Astrofisica de Andalucia (CSIC), Glorieta de Astronomia, E-18008 Granada, Spain

\section{B. Gendre}

University of Virgin Islands, 2 John BrewerÕs Bay, St Thomas, VI 00802, USA; Etelman Observatory, St Thomas, VI 00802, USA

\section{Malesani}

Dark Cosmology Centre, Niels Bohr Institutet, University of Copenhagen, Juliane Maries Vej 30, DK-2100 Copenhagen Denmark

\section{A. Rossi Istituto Astrofisica Spaziale e Fisica Cosmica di Bologna (INAF), Via P. Gobetti 101, I-40129 Bologna, Italy}




\title{
N. Gehrels, E. Troja
}

NASA Goddard Space Flight Center, Greenbelt, Maryland, MD 20771, USA

\section{Piro}

IAPS-INAF, Via Fosso del Cavaliere 100, 00133, Rome, Italy

\section{Boër}

CNRS-ARTEMIS, Boulevard de lÕObservatoire, CS 34229, F-06304 Nice Cedex 4, France

\section{G. Stratta}

Department of Physics, University of Urbino, V. S. Chiara 27, I-61029 Urbino, Italy

\begin{abstract}
Gamma-ray Burst 130427A had the largest fluence for almost 30 years. With an isotropic energy output of $8.5 \times 10^{53} \mathrm{erg}$ and redshift of 0.34 , it combined a very high energy release with a relative proximity to Earth in an unprecedented fashion.

Sensitive X-ray facilities such as XMM-Newton and Chandra detected the afterglow of this event for a record-breaking baseline of 90 Ms. We show the X-ray light curve of GRB 130427A of this event over such an interval. The light curve shows an unbroken power law decay with a slope of $\alpha=1.31$ over more than three decades in time.

In this presentation, we investigate the consequences of this result for the scenarios proposed to interpret GRB 130427A and the implications in the context of the forward shock model (jet opening angle, energetics, surrounding medium). We also remark the chance of extending GRB afterglow observations for several hundreds of Ms with Athena.
\end{abstract}

XII Multifrequency Behaviour of High Energy Cosmic Sources Workshop

12-17 June, 2017

Palermo, Italy

\footnotetext{
*Speaker.

${ }^{\dagger}$ A footnote may follow.
} 


\section{Introduction}

Gamma-ray Bursts (GRBs) are the brightest explosions in the Universe (Kumar \& Zhang 2015), being able to release up to $\sim 10^{55}$ erg in the $\gamma$-ray band within few hundreds seconds. They are followed by the so-called "afterglow", which is a slowly fading emission detected across the whole electromagnetic spectrum. The most popular theoretical model used to explain the afterglow is the so called "forward shock" (FS; Sari, Piran \& Narayan 1998). In this model, the ultrarelativistic ejecta of the explosion drive a shockwave in the circumburst medium; this shockwave accelerates the electrons of the medium which, in turn, re-emits the energy as synchrotron radiation. The FS model predicts the flux at any wavelength and epochs on the basis of few parameters, namely the kinetic energy $E_{\mathrm{K}}$, the fraction of energy in radiating electrons and magnetic field $\varepsilon_{\mathrm{e}}$ and $\varepsilon_{\mathrm{B}}$, the index of the power law energy distribution of electrons $p$, and the density and profile of the circumburst medium. By investigating the afterglow, we can check whether it can be explained by the FS model and whether the parameters inferred are realistic. In this article, we deal with GRB 130427A, the event with the largest fluence in the last 29 years. Assuming isotropy, in the $\gamma$-ray band this burst released $E_{\gamma, \text { iso }}=8.5 \times 10^{53} \mathrm{erg}$ (Perley et al. 2014) at redshift $z=0.34$. Only $\lesssim 3 \%$ of GRBs have a larger $E_{\gamma, \text { iso }}$, and $\lesssim 4 \%$ of them occur at lower redshift. Thus, GRB 130427A represents an unique chance to study a very bright event and test the FS model in detail. In the following, we use the convention $F_{v} \propto v^{-\beta} t^{-\alpha}$, where $F_{v}$ is the flux density, $v$ and $t$ are frequency and time since trigger respectively. Errors are at $68 \%$ confidence level (CL), unless otherwise specified, while $N_{x}=N \times 10^{x}$.

\section{Observations and Results}

Our dataset is constituted by X-ray observations carried out by Swift X-ray Telescope (XRT; Burrows et al. 2005), XMM-Newton and Chandra. XRT observed GRB 130427A until October 2013. XMM-Newton carried out observations in May, June and December 2013, in May and December 2015 (PIs: De Pasquale, Boer). Further, we used publicly available Chandra observations (PI: Fruchter) taken in February and June 2014, January 2015, January 2016. Even the last XMM and Chandra observations, taken $\simeq 90 \mathrm{Ms}$ after the trigger, led to a significant detection. These are the latest known detections of an X-ray afterglow of a cosmological GRB. Reduction and analysis of the data were carried out as described in De Pasquale et al. (2016). The derived 90 Ms X-ray light-curve of GRB 130427A is shown in Figure 1. When analyzing the light-curve, we consider only observations taken after $47 \mathrm{ks}$, because we are interested in the consistency of late time data with the proposed scenarios. We find that a simple power law model, i.e. flux $F \propto t^{-\alpha}$, fits the data adequately, with $\chi^{2} /$ d.o.f. $=79.3 / 68$. The best fit $\alpha=1.312 \pm 0.007$. Power law models with one or more breaks do not yield significant improvements to the fit.

\section{Discussion}

The standard FS model predicts phenomena occurring over long time scales in GRB afterglows. Some of them are: 


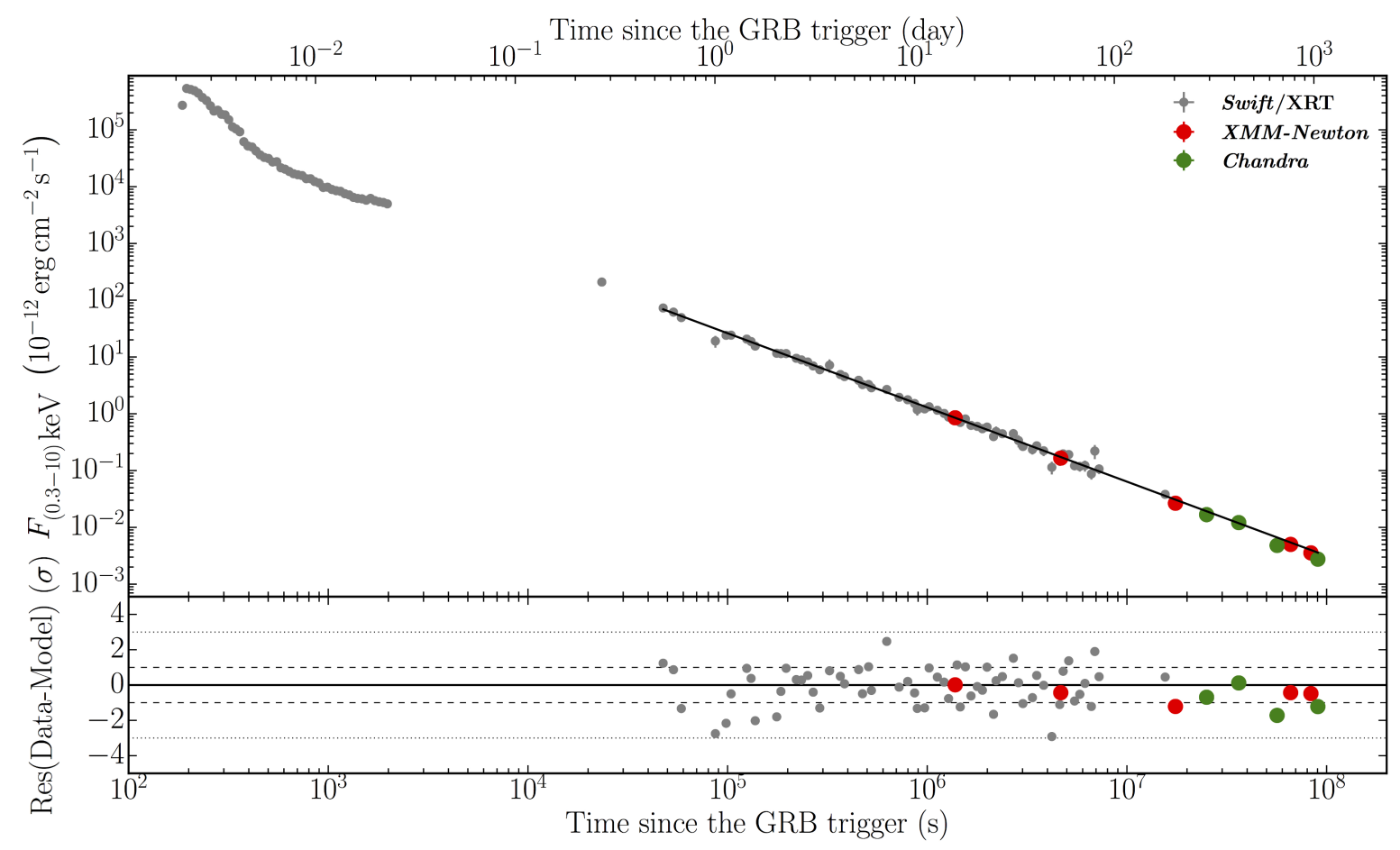

Figure 1: GRB 130427A X-ray afterglow over 90 Ms. The solid line shows our best fit model. Adapted from De Pasquale et al. (2016)

- Jet break (Zhang \& MacFadyen 2009) i.e. a steepening of the light curve down to a slope $\alpha \simeq p$; this phenomenon is interpreted as presence of collimated ejecta. The Lorentz factor $\Gamma$ of the ejecta is supposed to decrease in time because these sweep circumbust medium. When $\Gamma^{-1} \sim \theta_{\text {jet }}$, where $\theta_{\text {jet }}$ is the semi-opening angle of the ejecta, the observer will see the entire emitting surface and no further radiation previously beamed away will be detected. This feature causes a steep drop of the afterglow light-curve.

- Change of physical parameters of the shock emission. There is no reason to think that, over a long period of time and substantial changes of the dimension of the ejecta, the physical parameters may not evolve.

- Change of the density profile of the circumburst medium. Long GRBs like 130427A are linked to the demise of massive stars. These objects produce strong stellar winds; thus it is expected that the GRB ejecta will initially move into an environment with wind density profile. However, they will eventually reach a distance at which a medium of constant density profile is present.

However, these phenomena will show up differently in the afterglow light curves depending on the environment. In this respect, different groups have adopted different environments in their modeling of GRB 130427A afterglow. Laskar et al. (2013; henceforth L13) and Perley et al. (2014; 
P14) have assumed a free stellar wind environment, in which density $\rho$ decreases with radius $r$ as $\rho=A r^{-2}$. Kouveliotou et al. (2013; K13) and van der Horst et al. (2014,V14) have settled on a non-standard stellar wind, in which $\rho=A r^{-1.4}$ and $\rho=A r^{-1.7}$ respectively. Finally, Maselli et al. 2014 (M14) have assumed a constant density medium (as in the interstellar medium; ISM). All these authors have modeled the afterglow emission with data up to a few months after the trigger. We will test whether their modeling can still hold and explain the X-ray data extending for $\simeq 3$ years.

\subsection{Models in $r^{-2}$ stellar wind}

In a free wind region, the radius $R$ reached by the ejecta will be

$$
R=4.8 E_{\mathrm{K}, \mathrm{iso}, 54}^{1 / 2} A_{\star,-1}^{-1 / 2} t_{6}^{1 / 2} \mathrm{pc}
$$

(Chevalier \& $\operatorname{Li} 2000$ ) where $A_{\star}$ is the wind density normalization for a mass loss rate of $10^{-5} \dot{M}_{\odot} \mathrm{yr}^{-1}$ for the progenitor of the GRB, and the pedex "iso" means that we are considering isotropic energy. According to the standard picture of a stellar wind bubble (Weaver et al. 1977), the region of free stellar wind of radius $R_{1}$ is contained within a region of shocked stellar wind where density is constant. Thus, the expanding ejecta will unavoidably have to enter into the latter region. When this happens, the FS model predicts a change in afterglow decay slope if the observing band is between the synchrotron peak frequency $v_{\mathrm{m}}$ and cooling frequency $v_{\mathrm{c}}$, as is assumed by both L13 and P14. In an ISM environment, the decay slope is expected to be $\alpha=3 / 4 p-3 / 4$. L13 and P14 assume $p \simeq 2.2$, thus the predicted slope is $\alpha=0.9$. However, we derive a $95 \%$ lower limit of $63 \mathrm{Ms}$ for any flattening to $\alpha=0.9$ of GRB 130427A X-ray light-curve. Knowing this limit and the values of $A_{\star}$ and kinetic energy derived by L13 and P14 ${ }^{1}$, we can use equation 3.1 to find a lower limit on $R_{1}$. We determine $R_{1}>120 \mathrm{pc}$ and $R_{1}>57 \mathrm{pc}$ for P14 and L13, respectively. The radius of the free stellar wind region is given by $R_{1} \simeq \dot{M}_{-5}{ }^{1 / 3} n_{0,2}^{-1}$ pc (Fryer et al. 2006), where $\dot{M}$ is the mass loss rate in $M_{\odot} \mathrm{yr}^{-1}$ and $n_{0}$ is the density of the pre-existing material in which the stellar wind bubble was blown. Assuming a standard $\dot{M}_{-5}=1$, the previous lower limits on $R_{1}$, we find upper limits $n_{0}<1.5 \times 10^{-4}$ and $n_{0}<6 \times 10^{-4} \mathrm{~cm}^{-3}$. These densities are unrealistic, because they are too low for star forming regions where the massive progenitors of GRBs are expected to be located. HII region surveys (Hunt \& Hirashita 2009, Peimbert \& Peimbert 2013) yield densities $\gtrsim 1 \mathrm{~cm}^{-3}$. According to the numerical simulations of Sharma et al. (2014) and Yadav et al. (2016), star clusters with more than $\sim 10^{4} \mathrm{OB}$ stars could produce "super blubbles" in which density decreases as $\rho \simeq r^{-2}$ for $\sim 100$ pc. However, even the most massive star clusters in the Local Group may not contain so many massive stars (Beck 2015). We also note that the afterglow of GRB 130427A is not located outside the optical image of its host galaxy (Levan et al. 2014). Moreover, the lowionisation lines of the optical spectrum show no signature of galactic winds (Khrühler et al., priv. comm.). Thus, it is unlikely that the burst occurred in a low density extragalactic environment, or in a very large wind environment extending above the galactic disk.

\footnotetext{
${ }^{1} A=0.003$ in both articles, while $E_{\mathrm{K}, \text { iso, } 54}$ is 0.3 and 0.07 for P14 and L13 respectively.
} 


\subsection{Models in non-standard wind environs}

$\mathrm{K} 13$ assumptions are pure synchrotron emission (i.e. no inverse Compton), $p=2.34$ and $\mathrm{X}$ ray band between $v_{m}$ and $v_{c}$. We modeled the $\mathrm{X}$-ray afterglow imposing the conditions above and the observed X-ray flux and we derived $E_{\mathrm{K} \text {,iso }} \simeq 10^{54} \mathrm{erg}$ and $A \simeq 10^{-3} \mathrm{~g} \mathrm{~cm}^{-1.6}$. This density normalization value implies a very thin wind, with densities $\sim 10^{-7} \mathrm{~cm}^{-3}$ at $20 \mathrm{pc}$ from the explosion site. As in the previous scenario, we need a very big stellar wind radius, $R \gtrsim 170 \mathrm{pc}$. Thus, even the scenario proposed by K13 runs into the problem of a too low density of the pre-existing material.

V14 scenario presumes a two-component jet with a narrow and a wide component. The afterglow is the sum of the emission of the two components. V14 assumes parameters within a wide range and that the physical parameters, such as $\varepsilon_{e}$ and $\varepsilon_{B}$, evolve with time in a different fashion depending on whether they characterize the wide or the narrow component. We believe that this model could indeed account for the late X-ray data, but it may achieve that by several indeterminacies of its parameters; see De Pasquale et al. (2016) for more details.

\subsection{ISM model with early jet break and evolving parameters}

M14 scenario consists of constant density medium and an early jet break at $37 \mathrm{ks}$. After jet breaks, the decay slopes is supposed to be steep; however M14 postulates evolving parameters: $\varepsilon_{\mathrm{e}}=$ $0.027 \times(\mathrm{t} / 0.8 \mathrm{~d})^{0.6}, \varepsilon_{\mathrm{B}}=10^{-5} \times(\mathrm{t} / 0.8 \mathrm{~d})^{0.5}, \xi=(\mathrm{t} / 2 \mathrm{~d})^{-0.8}$, where $\xi$ is the fraction of accelerated, emitting electrons. With this evolution, the decay slope of the X-ray afterglow can be $\alpha \simeq 1.3$ even in a post jet-break regime. However, M14 used data up to $\simeq 4.2 \mathrm{Ms}$ after the trigger, while our data extend $\simeq 20$ times as long. The parameter $\varepsilon_{\mathrm{e}}$ is not supposed to be larger than $1 / 3$, the so-called "equipartition" value. With the evolution assumed by M14, this value would be reached already at $\simeq 4.5$ Ms. Moreover, $\xi \simeq 0.001$ at $\simeq 90 \mathrm{Ms}$, and it not clear why we would be accelerating only such a tiny fraction of electrons. Overall, we conclude that the ISM scenario with evolving parameters and early jet break is weakened by our new dataset.

\subsection{A basic ISM scenario?}

Could the FS model, in the basic ISM form, explain the behaviour of GRB 130427A X-ray afterglow? To answer this question, we can first take a look at the decay and spectral indices. The FS scenario predicts relations between these two indices that depend on the position of the observing frequency, the medium density profile, and the kind of expansion, jetted or spherical. In the case of X-ray emission of GRB 130427A, the relevant cases are $v_{\mathrm{X}}<v_{\mathrm{c}}$ and $v_{\mathrm{X}}>v_{\mathrm{c}}$, where $v_{\mathrm{X}}$ is the frequency of the $\mathrm{X}$-ray band, and spherical expansion. In the former case, $\alpha=3 / 2 \beta$, while in the latter we should have $\alpha=(3 \beta+5) / 8^{2}$. Since we find $\alpha=1.312 \pm 0.007$ and $\beta=0.79 \pm 0.03$, the relation for the $v_{\mathrm{X}}>v_{\mathrm{c}}$ is not satisfied, while $v_{\mathrm{X}}<v_{\mathrm{c}}$ is satisfied within $\simeq 2.5 \sigma$. The next step to check whether this FS model is viable is examine the required parameters, especially energy. The total energy corrected for beaming effects is $E_{\text {tot,corr }}=\left(E_{\gamma, \text { iso }}+E_{\mathrm{K} \text {,iso }}\right) \times f_{\mathrm{b}}$, where $f_{\mathrm{b}}=\theta_{\text {jet }}^{2} / 2$ is the "beaming factor" that takes into account the possibility that the ejecta are collimated within an semi-opening angle $\theta_{\text {jet }}$. It is possible to derive or constrain the value of $\theta_{\text {jet }}$ with the formula

\footnotetext{
${ }^{2}$ For $p>2$ the expression is $\alpha=3 / 2 \beta-1 / 2$. The above expression is valid for $p<2$ which would occur for $v_{\mathrm{X}}>v_{c}$ for the value of $\beta$ we find.
} 


$$
\theta_{\text {jet }}=0.12\left(\frac{t_{\text {jet }, \mathrm{d}}}{1+z}\right)^{3 / 8}\left(\frac{E_{\mathrm{K}, \text { iso }, 53}}{n}\right)^{-1 / 8} \mathrm{rad}
$$

(Zhang \& MacFadyen 2009) where $t_{\text {jet,d }}$ is the jet break time, expressed in days, and $n$ is the density of the environment. Fitting the X-ray light curve, we derive a 95\% CL lower limit for $t_{\text {jet }}=62$ Ms. After some algebra, we find that this lower limit on $t_{\text {jet }}$ implies lower limits $\theta_{\text {jet }} \geq 0.47 \mathrm{rad}$ and $E_{\text {tot,corr }} \geq 1.2 \times 10^{53} \mathrm{erg}$. Assumptions on $n$ and on the ratio between $E_{\mathrm{K}}$ and $E_{\gamma}$ are required to calculate the lower limits on $\theta_{\text {jet }}$ and $E_{\text {tot,corr }}$; we made those assumptions that minimize the value of the opening angle and energy ${ }^{3}$. Thus, the lower limit energy requirement is very high; in realistic conditions, a black hole of a few tens of solar masses would be required to produce the output.

A variant of this scenario, which manages to reduce the energy requirement, is the off-axis case. If the Earth is at an angle $\theta_{\text {obs }}$ with respect to the jet axis, the steepening in the light curve will be visible only when the observer sees emission from the "far end" of the outflow. In Eq. 3.2, one must replace $\theta_{\text {jet }}$ with $\theta_{\text {jet }}+\theta_{\text {obs }}$. Thus, $\theta_{\text {jet }}$ is reduced, and as a consequence $E_{\text {tot,corr }}$ decreases as well. We find that for $\theta_{\mathrm{obs}}=0.4 \theta_{\text {jet }}$, the lower limit on total energy corrected for beaming is $E_{\text {tot,corr }} \gtrsim 6.5 \times 10^{52} \mathrm{erg}$. While this is still a rather large amount, it is half the value required in the simple on-axis case. We point out, however, that this ISM scenario needs testing against data in bands other than the X-ray one.

\section{Conclusions}

We presented XMM-Newton and Chandra observations of the afterglow of the exceptionally luminous GRB 130427A, which took place up to $90 \mathrm{Ms}$ from the trigger. This is the longest follow up for the X-ray afterglow of a cosmological burst. We found that the flux of the source shows a simple power law decay with slope $\alpha=1.312 \pm 0.007$ till very late epochs; no jet break or other changes of slope are detected. We tested the durability of scenarios built on data up to $\sim 100$ days from the trigger and based on the standard, forward shock model for GRB afterglows. The scenarios in free stellar wind we examined (P14, L13) require densities of the pre-existing medium which are far too low for star forming regions. The scenarios in non-standard stellar wind either involve a too low density environment (K13) as well, or evolving and unconstrained parameters (V14). The ISM model proposed by M14 assumes an early jet break with evolving physical parameters, but it is hard to keep the decay slow for $90 \mathrm{Ms}$.

We found that the simple, ISM scenario with on-axis observer requires $E_{\text {tot,corr }} \gtrsim 1.2 \times 10^{53} \mathrm{erg}$. However, an off-axis variant of the same scenario could still explain observations with $E_{\text {tot,corr }} \gtrsim 6.5 \times$ $10^{52} \mathrm{erg}$.

The worst case scenario for future observations of GRB 130427A X-ray afterglow is that a jet break occurs now. If that happened, the predicted flux at the launch of the Athena mission (2028, Nandra et al. 2013) would be around $10^{-16} \mathrm{erg} \mathrm{cm}^{-2} \mathrm{~s}^{-1}$; see Figure 2. We point out that a source with this flux would still be detectable by Athena. Thus, by means of the ESA flagship X-ray observatory, we will be able to extend the time coverage of GRB 130427A by one order of

\footnotetext{
${ }^{3}$ To see the full derivation refer to De Pasquale et al. (2016).
} 
GRB 130427A X-ray LC

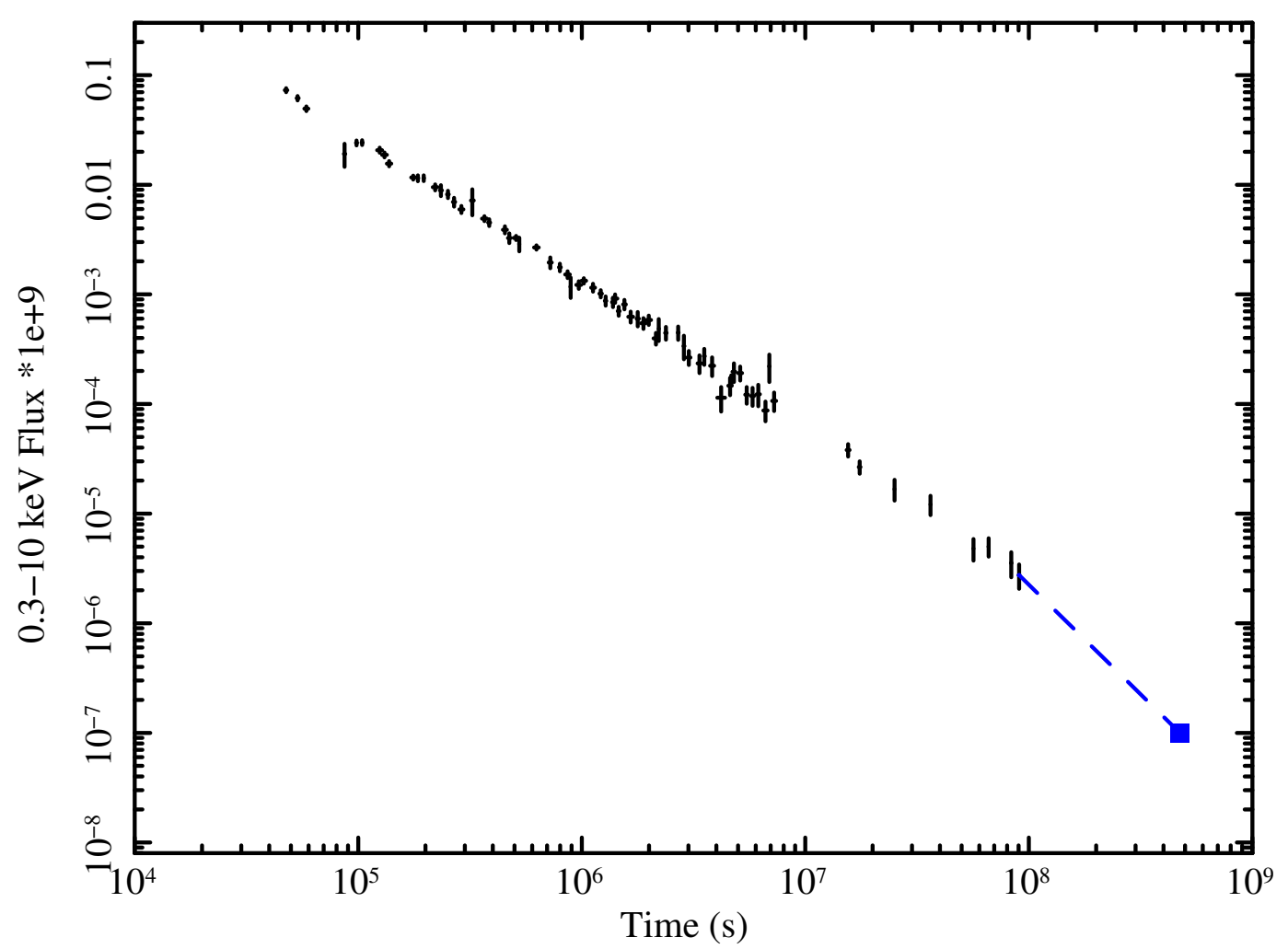

Figure 2: GRB 130427A X-ray afterglow light curve extended to 2028 in the worst-case scenario for the flux (dashed blue line). At that epoch, the afterglow would still be detectable by the ESA Athena mission.

magnitude. If we found that no change of slope has occurred by then, the problems for the FS model we have highlighted so far would be further exacerbated to the point that this model should be abandoned.

This work was supported by Scientific Research Project Coordination Unit of Istanbul University, project number: BEK-2017-25641.

\section{References}

[1] S. Beck, The youngest globular clusters, IJMPD 24 (2015) 1530002 [ast ro-ph/1412 . 0769].

[2] D. Burrows, J. Nousek, J. Kennea, et al., The Swift X-Ray Telescope, SSRv 120 (2005) 165 [astro-ph/0508.071]. 
[3] R. Chevalier \& Z.-Y. Li, Wind Interaction Models for Gamma-Ray Burst Afterglows: The Case for Two Types of Progenitors, ApJ 536 (2005) 195 [astro-ph/9908.272].

[4] M. De Pasquale, M. Page, D. A. Kann, et al., The 80 Ms follow-up of the X-ray afterglow of GRB 130427A challenges the standard forward shock model, MNRAS 462 (2016) 1111 [astro-ph/1602.04158].

[5] C. Fryer, G. Rockefeller, \& P. Young, The Environments around Long-Duration Gamma-Ray Burst Progenitors, ApJ 647 (2006) 1269 [astro-ph/ 0604 . 432].

[6] L. Hunt \& H. Hirashita, The size-density relation of extragalactic H II regions, A\&A 507 (2009) 1327 [astro-ph/0910.2804].

[7] C. Kouveliotou, J. Granot, J. Racusin, et al., NuSTAR Observations of GRB 130427A Establish a Single Component Synchrotron Afterglow Origin for the Late Optical to Multi-GeV Emission, ApJ 779 (2013) 1 [astro-ph/1311.5245]

[8] P. Kumar \& B. Zhang, The physics of gamma-ray bursts \& relativistic jets, PhR 561 (2015) 1 [astro-ph/1410.0679].

[9] T. Laskar, E. Berger, B. Zauderer, et al., A Reverse Shock in GRB 130427A, ApJ 776 (2013) 119 [astro-ph/1305.2453].

[10] A. Levan, N. Tanvir, A. Fruchter, et al., A Hubble Space Telescope Observations of the Afterglow, Supernova, and Host Galaxy Associated with the Extremely Bright GRB 130427A, ApJ 792 (2014) 115 [astro-ph/1307.5338].

[11] A. Maselli, A. Melandri, L. Nava, et al., GRB 130427A: A Nearby Ordinary Monster, Science 343 (2014) 48 [astro-ph/1311.5254].

[12] K. Nandra, D. Barret, X. Barcons, et al., The Hot and Energetic Universe: A White Paper presenting the science theme motivating the Athena+ mission, (2013) astro-ph/1306.2307.

[13] A. Peimbert \& M. Peimbert, Densities, Temperatures, Pressures, and Abundances Derived from O II Recombination Lines in H II Regions and their Implications, A\&A 778 (2013) 89 [astro-ph/1310.0089].

[14] D. Perley, S. Cenko, A. Corsi, et al., The Afterglow of GRB 130427A from 1 to $10^{16} \mathrm{GHz}$, ApJ 781 (2014) 37 [astro-ph/1307.4401]

[15] R. Sari, T. Piran \& R. Narayan, Spectra and Light Curves of Gamma-Ray Burst Afterglows, ApJL 497 (1998) 17 [astro-ph/9712.005].

[16] P. Sharma, A. Roy, B. Nath, et al., In a hot bubble: why does superbubble feedback work, but isolated supernovae do not?, MNRAS 443 (2014) 3463 [astro-ph/1402 . 6695].

[17] A. van der Horst, Z., Paragi, A. G. de Bruyn, et al., A comprehensive radio view of the extremely bright gamma-ray burst 130427A, MNRAS 444 (2014) 3151 [astro-ph/1 404 . 1945]

[18] N. Yadav, M. Dipanjan, P. Sharma, et al., How multiple supernovae overlap to form superbubbles, MNRAS 465 (2017) 1720 [astro-ph/1603. 00815 ]

[19] T. Weaver, R. McCray, J. Castor, et al., Interstellar bubbles. II - Structure and evolution, ApJ 218 (1977) 377.

[20] W. Zheng \& A. MacFadyen, The Dynamics and Afterglow Radiation of Gamma-Ray Bursts. I. Constant Density Medium, ApJ 698 (2009) 1261 [astro-ph/0902 . 2396]. 


\section{DISCUSSION}

DANIELE FARGION: I want to remind that the GRBs by precessing thin jet have a foreseen flux decay $\propto\left(\frac{t}{3 \times 10^{4} \mathrm{~s}}\right)^{-1}$ or somehow lower power law. The rare persistence is due to the uncommon on-axis beaming, and its late decay several years or century later may interface GRBs with late SGRs (Fargion 1999, AA, 138, 507; Fargion \& Oliva, arXiv-160500177).

MASSIMILIANO DE PASQUALE: Thank you for your comment. 